\author{
Monica E. Busse \\ Gareth Hughes \\ Charles M. Wiles \\ Anne E. Rosser
}

\section{Use of hand-held dynamometry in the evaluation of lower limb muscle strength in people with Huntington's disease}

Received: 13 December 2007

Received in revised form: 24 March 2008

Accepted: 3 April 2008

Published online: 2 August 2008

Dr. M. E. Busse $(\varangle) \cdot$ G. Hughes

Dept. of Physiotherapy

School of Healthcare Studies

Cardiff University

Ty Dewi Sant, Heath Park

Cardiff, CF14 4XN, UK

E-Mail: busseme@cardiff.ac.uk

C. M. Wiles · A. E. Rosser

Dept. of Neurology

School of Medicine

Cardiff University Heath Park

Cardiff CF14 4XN, UK

\section{A. E. Rosser}

Brain Repair Group

School of Biosciences

Cardiff University

Museum Avenue

Cardiff CF15 8DQ, UK
Abstract Purpose Sub-clinical muscle involvement, including myopathic changes and mitochondrial dysfunction of skeletal muscle, has been reported in people with Huntington's disease (HD). Muscle strength was evaluated using a hand-held dynamometer. Reliability and validity in people with HD were determined. Method Isometric muscle strength of 6 lower limb muscle groups was measured in 20 people with HD and matched healthy controls. People with HD were evaluated with the Unified Huntington's Disease Rating Scales (UHDRS). Within session reliability using intra-class correlation coefficients (ICC) was calculated. Discriminant and convergent validity was also evaluated. Results UHDRS motor scores of people with HD ranged from 28 to 80 . Reliability of strength testing was excellent (ICC 0.86 to 0.98 ). People with HD had on average about half the strength of healthy matched controls. UHDRS motor scores and strength scores were significantly correlated (convergent) providing a further indication of validity of strength testing. Conclusions The hand-held dynamometer is a reliable and valid measurement tool to detect strength differences between people with HD and a matched control group. There is significant reduction in lower limb muscle strength in HD which does not appear to have been described previously.

Key words muscle strength . muscle weakness $\cdot$ Huntington's Disease $\cdot$ dynamometry $\cdot$ reliability

\section{Introduction}

Hand-held dynamometry has been used to assess the muscle strength of people with neurological disorders $[2,13,17,33-35]$; use has not however been reported in people with Huntington's disease (HD). Huntington's disease is a progressive, neurodegenerative disease that results in symptoms of movement disorder. Although clinical muscle weakness is not a prominent feature in the pre-symptomatic or early symptomatic stages of the with myopathic change on biopsy [3] and biochemical
evidence of mitochondrial dysfunction of skeletal mus- cle has been reported [22]. Given the known evidence of early muscle mitochondrial dysfunction (e.g. delayed phosphocreatine recovery following aerobic or ischaemic exercise even in presymptomatic individuals) [24], it was of interest to systematically examine muscle strength in the disorder. Weakness, if found, might be either due to a disorder of the control of neural excitation processes due to CNS or peripheral nerve disorder or due to disordered muscle excitation, activation or energy exchange processes within muscle or due to reduced muscle mass (or a combination of these).

Lower limb muscle weakness may limit functional abilities in people with Huntington's disease (HD). In addition significant reductions of muscle strength may 
occur below the manual testing threshold for weakness even in individuals who can maintain routine activities of daily living. The incidence of muscle weakness in this group needs to be established with the use of objective reliable and valid measurements and comparison with appropriate control subjects.

Reliability refers to whether or not a measure can be repeated accurately by different assessors (inter-rater) and by a single rater (intra-rater) [4]. Documentation of any change of muscle strength as a result of an intervention i.e. before and after treatment should incorporate knowledge of reliability and take associated variability into account. Any measured difference will include some variability that could be due to the natural variance of the participant or the measuring process or both.

Reliability is established for the use of hand held dynamometers in people with neurological disorders provided that a specific protocol and trained raters are used to perform the testing $[8,34]$; however this requires confirmation in people with HD.

Knowledge of the reliability and associated variability of tests of muscle strength is important to be able to judge whether true changes or differences between populations exist whilst knowledge of validity is required to make inferences regarding the clinical implications of any particular test. Discriminant and convergent validity are both considered subcategories of construct validity. Tests that are required to detect differences on the same construct should discriminate in known groups; this is often called known group method [10]. For example people with HD may be expected to demonstrate differences in muscle strength relative to a healthy matched control group as they are known to be clinically different.

The best known and best validated clinical assessment tool for HD is the Unified Huntington's Disease Rating Scale (UHDRS) [20]. The UHDRS comprises a series of semi-quantitative clinical scales to assess motor function, cognitive function, behavioural abnormalities, and functional capacity. Tests that measure similar constructs should converge, for example one might expect higher UHDRS motor scores in the presence of muscle weakness as they indicate greater impairment for the motor assessment (UHDRS motor maximum possible score $($ worst $)=124)$. The UHDRS functional scale comprises three components (Functional Assessment Scale (FAS) (minimum possible score (worst) $=0$ ), the Independence Scale (IS) (lowest possible score (worst) $=5$ ), and the Total Functional Capacity (TFC) (lowest possible score (worst) $=0)$ ). It is feasible that lower functional scores would be expected in the presence of muscle weakness [15, 27]. Knowledge of relations between routinely used current clinical measures such as the UHDRS scores can be useful in understanding disease progression and the relative information relating to function that can be gained by utilising strength testing in the specific population.
The aim of this study was to evaluate lower limb strength in people with HD using hand-held dynamometry and to assess reliability, related variability and the construct validity (represented by any relationships with standard clinical measures in HD).

\section{Method}

\section{Subjects}

A convenience sample of 20 symptomatic people with HD and 20 healthy control subjects were recruited to this study. Inclusion criteria for the people with HD were that they had a confirmed diagnosis of $\mathrm{HD}$ and were able to provide informed consent. They were required to be able to walk a distance of 10 metres with or without assistance. Inclusion criteria for the healthy subjects were that they had no peripheral injury or other condition that could impact on muscle strength testing. Ethical approval for the study was obtained from the local research ethics committee. All subjects provided informed consent before participation. All recruited subjects participated in all aspects of the study.

\section{Data collection}

Demographic data of age (years), height $(\mathrm{cm})$ and weight $(\mathrm{kg})$ were obtained. Scores on the motor section of the Unified Huntington's disease Rating Scale (UHDRS motor), the Total Functional Capacity (TFC), the Functional Assessment Scale (FAS) and the Independence Scale (IS) were recorded for the people with HD.

\section{Strength testing}

The Powertrak hand-held dynamometer (JTech Medical, Utah, USA) was used to evaluate muscle strength in this study. Muscle strength was defined "as the maximal voluntary force that the subjects were able to exert on the environment under the specific testing conditions" [7]. Each subject performed three maximum voluntary isometric instantaneous contractions for each requested test movement. Subjects were instructed to build the force up over 2 seconds and then hold that maximum for another 3 seconds. All test movements and positions were standardised using a pragmatic approach that minimised the required alteration of body positions for testing whilst allowing the use of simple instructions (Table 1). The right side was tested before the left side systematically. Two assessors experienced in the use of hand-held dynamometry conducted all the assessments; inter-rater reliability was established prior to the start of the study.

A strength index for each of the three repetitions per muscle group and thereafter the best score [16] for each muscle group was used to calculate a maximal strength index [36]. The method combines the contribution from all muscle groups into a single index. Using the healthy control subject data $(n=20)$ the strongest muscle group, knee extension, was allocated a factor of 1 . The factors for all the other muscles were calculated as the ratio of average knee extensor strength to the average strength of the muscle tested [36]. Scores for each muscle group were then multiplied by the relevant factor and summed to produce the final strength index for the right and left sides separately. Factors for knee extension, hip extension, hip abduction, knee flexion, ankle dorsi flexion and ankle plantar flexion were $1,1.26,1.43,1.55,1.31$ and 1.81 respectively.

In order to represent muscle weakness in terms of standard deviation units relative to the mean of a healthy matched control group [1], a standardised score (z-score) for each variable for the HD group was calculated as the difference between the HD group score and the 
Table 1 Standardised testing positions for lower limb muscle groups

\begin{tabular}{lll}
\hline Muscle action & Load cell position & Starting position \\
\hline $\begin{array}{l}\text { Hip extension } \\
\text { Hip abduction }\end{array}$ & $\begin{array}{l}10 \mathrm{~cm} \text { proximal to knee joint, in line with the middle of the knee joint } \\
\text { In line with the knee joint, mid-lateral aspect of joint }\end{array}$ & $\begin{array}{l}\text { Standing facing the plinth, both lower limbs in neutral extension } \\
\text { (plinth can be used for stabilisation when balancing on single leg) }\end{array}$ \\
\hline Knee extension & $\begin{array}{l}\text { Immediately superior to lateral malleolus of the ankle, anterior } \\
\text { surface in line with the middle of ankle joint } \\
\text { Immediately superior to lateral malleolus of the ankle, posterior } \\
\text { Surface in line with the middle of ankle joint }\end{array}$ & Sitting on plinth: hips and knees flexed to $90^{\circ}$, hands resting in lap \\
\hline Ankle dorsiflexion & On the dorsum of the foot in line with second metatarsal of the foot & $\begin{array}{l}\text { Sitting: hip flexed and knee flexed to } 90^{\circ} \text {, ankle in neutral. Hands } \\
\text { resting in lap }\end{array}$ \\
\hline Ankle plantar flexion & $\begin{array}{l}\text { On the plantar surface of the foot in line with second metatarsal of } \\
\text { the foot }\end{array}$ & $\begin{array}{l}\text { Sitting: hips and knees flexed to } 90^{\circ} \text { and ankle in neutral. Hands } \\
\text { resting in lap }\end{array}$ \\
\hline
\end{tabular}

control group average divided by the standard deviation of the control group.

\begin{abstract}
Data analysis
The Statistical Package for the Social Sciences (SPSS) Version 12 (SPSS Inc., Chicago, USA) was used for all data analyses. Within session reliability of the three maximal voluntary contraction attempts in both groups (for each muscle group and each side) was assessed with a two-way mixed model intra class correlation coefficient (ICC) with measures of consistency (ICC 3,1) [26]. Single measures and average measures ICC values [26] were calculated to determine whether one repetition (i.e. the best of three attempts [16]) or the average of three repetitions was required for reliable testing. As ICC scores are susceptible to sample heterogeneity, the average coefficient of variation $(\mathrm{CoV})$, calculated as the standard deviation of the measures expressed as a percentage (\%) of the mean for the three repetitions [5] was calculated to provide an estimate of any associated error and to allow for assessment of differences in variability between groups. Difference data were assessed for normality using $Q-Q$ plots and histograms. Differences between groups were assessed using independent t-tests for interval data and chi square tests for categorical data or where data were not normal the appropriate non-parametric equivalent test was utilised. Discriminant validity was assessed using the known-groups method [10] where differences between people with HD and healthy subjects were assessed for each of the calculated variables.

Convergent validity of muscle strength testing was assessed by examining the correlations (spearman's correlation coefficients) between UHDRS scores and a) best of the three maximal strength attempts for individual lower limb muscle groups (right and left) and b) a strength index (SI) calculated for each limb [36].
\end{abstract}

\section{Sample sizes}

The sample sizes were considered sufficient to both characterise reliability and allow for more in-depth between and within group comparisons. Twenty subjects tested three times will provide a power of $80 \%$ (alpha 0.05 ) [11] where reliability is moderate i.e. 0.6 [26] as measured by an intra-class correlation coefficient (ICC). When assessing for differences, 20 subjects per group is sufficient to detect a standardised difference of 0.9 [12] and 20 subjects is also sufficient to detect a correlation of 0.6 with a power of $80 \%$ and alpha of 0.05 (twotailed).

\section{Results}

\section{Subject characteristics}

All subjects participated in all parts of the study. The mean (SD) age (years), height $(\mathrm{cm})$ and weight $(\mathrm{kg})$ of people with HD $(n=20$; male $=13)$ was $51.7(7.6)$ years, $170.8(9.1) \mathrm{cm}$ and $75.8(14.1) \mathrm{kg}$ and of healthy controls $(\mathrm{n}=20$; male $=12)$ was $48.9(7.3)$ years, $173.3(10.5) \mathrm{cm}$ and $79.3(17.9) \mathrm{kg}$. There were no significant differences between groups for any of the matched variables of age, height, weight and gender.

Median (range) UHDRS, TFC, FAS and Independence scores were 48 (28-80), 6 (3-11), 17 (7-25) and 72.5 (5095) respectively.

\section{Strength scores}

Reliability using single measures ICC for all the muscle groups for each side was excellent and ranged from 0.86 to 0.96 in both groups; $95 \%$ confidence intervals (CI) were in similar ranges. Mean variability was mostly similar between groups and ranged from $9.1 \%$ to $15.6 \%$ in the HD group and from $5.4 \%$ to $13.9 \%$ in the healthy control group. Significantly greater variability relative to a healthy control group was identified for ankle dorsiflexion muscles. The leg strength index had the highest and most precise single measures reliability as well as the lowest variability, thus justifying further use of the single maximal score of best of three attempts to produce an MVC and strength index in this population [16]. The reliability indices (single and average measures) as well as $95 \%$ confidence intervals for the ICC scores and measures of variability for each strength score and the overall strength index for each limb for healthy control subjects and HD group are presented in Table 2.

People with HD were significantly weaker for all mus- 
cle groups and for both sides (right and left) relative to the matched healthy controlgroup. The maximal strength scores for the individual lower limb muscle groups (right and left), and maximal strength indices (SI) as well as $\mathrm{Z}$-scores for each limb for the HD group are presented in Table 3. On average the strength for the HD group was 1.55 standard deviations (SD) lower than what was seen in the matched healthy control group for the individual muscle groups and 1.8 SD less than controls for the SI.

Correlations between strength and functional scores for the HD group only are presented in Table 4. Hip ex- tension and abduction as well as ankle plantar flexion muscle strength were moderately correlated with UHDRS scores. Significant correlations with function were also identified for the strength indices.

\section{Discussion}

The findings presented indicate that the hand-held dynamometer can be considered a reliable measurement tool, reflects change in functional abilities in people with

Table 2 Reliability as measured by intraclass correlation coefficients (ICC) and average coefficients of variation (CoV). Differences between groups for muscles variability is also indicated $\left({ }^{*} p<0.05\right)$

\begin{tabular}{|c|c|c|c|c|c|c|c|}
\hline \multirow[t]{2}{*}{ Muscle action } & \multirow[t]{2}{*}{ Side } & \multicolumn{3}{|l|}{ HD group $(n=20)$} & \multicolumn{3}{|c|}{ Healthy control group $(n=20)$} \\
\hline & & $\begin{array}{l}\text { Single measures } \\
\text { ICC } \\
(95 \% \mathrm{Cl})\end{array}$ & $\begin{array}{l}\text { Average measures } \\
\text { ICC } \\
(95 \% \mathrm{Cl})\end{array}$ & $\begin{array}{l}\text { Mean (SD) } \\
\text { CoV \% }\end{array}$ & $\begin{array}{l}\text { Single measures } \\
\text { ICC } \\
(95 \% \mathrm{Cl})\end{array}$ & $\begin{array}{l}\text { Average measures } \\
\text { ICC } \\
(95 \% \mathrm{Cl})\end{array}$ & $\begin{array}{l}\text { Mean (SD) } \\
\text { CoV \% }\end{array}$ \\
\hline \multirow[t]{2}{*}{ Hip extension } & Right & $0.96(0.91-0.98)$ & $0.99(0.97-0.99)$ & $13.2(7.0)$ & $0.912(0.83-0.96)$ & $0.97(0.94-0.99)$ & $9.4(4.9)$ \\
\hline & Left & $0.98(0.95-0.99)$ & $0.99(0.98-0.99)$ & $9.1(5.2)$ & $0.93(0.86-0.97)$ & $0.98(0.95-0.99)$ & $8.9(3.9)$ \\
\hline \multirow[t]{2}{*}{ Hip abduction } & Right & $0.97(0.93-0.99)$ & $0.99(0.98-0.99)$ & $10.1(5.5)$ & $0.87(0.75-0.94)$ & $0.95(0.90-0.98)$ & $12.5(6.5)$ \\
\hline & Left & $0.97(0.93-0.99)$ & $0.99(0.98-0.99)$ & $10.5(7.2)$ & $0.92(0.84-0.97)$ & $0.97(0.94-0.99)$ & $12.4(6.4)$ \\
\hline \multirow[t]{2}{*}{ Knee extension } & Right & $0.86(0.73-0.94)$ & $0.95(0.89-0.98)$ & $13.6(17.2)$ & $0.90(0.80-0.95)$ & $0.96(0.92-0.98)$ & $8.9(6.0)$ \\
\hline & Left & $0.89(0.77-0.95)$ & $0.96(0.91-0.98)$ & $10.4(8.2)$ & $0.93(0.87-0.97)$ & $0.98(0.95-0.99)$ & $8.1(5.2)$ \\
\hline \multirow[t]{2}{*}{ Knee flexion } & Right & $0.87(0.75-0.95)$ & $0.95(0.90-0.98)$ & $13.8(11.7)$ & $0.93(0.85-0.97)$ & $0.97(0.95-0.99)$ & $9.7(5.8)$ \\
\hline & Left & $0.88(0.76-0.94)$ & $0.96(0.90-0.98)$ & $15.6(20.1)$ & $0.86(0.74-0.94)$ & $0.95(0.89-0.98)$ & $13.0(8.7)$ \\
\hline \multirow[t]{2}{*}{ Ankle dorsiflexion } & Right & $0.88(0.77-0.95)$ & $0.96(0.91-0.98)$ & $15.1(13.7)^{*}$ & $0.92(0.84-0.97)$ & $0.97(0.94-0.99)$ & $6.9(4.8)$ \\
\hline & Left & $0.94(0.88-0.97)$ & $0.98(0.96-0.99)$ & $11.9(13.9)^{*}$ & $0.96(0.92-0.98)$ & $0.99(0.97-0.99)$ & $5.4(3.3)$ \\
\hline \multirow[t]{2}{*}{ Ankle plantarflexion } & Right & $0.95(0.90-0.98)$ & $0.98(0.97-0.99)$ & $14.1(11.2)$ & $0.91(0.82-0.96)$ & $0.97(0.93-0.99)$ & $11.0(5.6)$ \\
\hline & Left & $0.96(0.92-0.98)$ & $0.99(0.97-0.99)$ & $10.5(7.9)$ & $0.89(0.78-0.95)$ & $0.96(0.91-0.98)$ & $13.9(7.0)$ \\
\hline \multirow[t]{2}{*}{ Leg strength } & Right & $0.98(0.96-0.99)$ & $0.99(0.98-0.99)$ & $7.5(4.9)$ & $0.96(0.92-0.98)$ & $0.99(0.97-0.99)$ & $4.7(2.1)$ \\
\hline & Left & $0.98(0.96-0.99)$ & $0.99(0.98-0.99)$ & $6.1(6.9)$ & $0.97(0.95-0.99)$ & $0.99(0.98-0.99)$ & $5.6(3.0)$ \\
\hline
\end{tabular}

Table 3 The maximal strength scores for the individual lower limb muscle groups and strength indices (SI) for healthy control group as well as z-scores for the HD group and $95 \% \mathrm{Cl}$ of differences between groups. Differences $\left.{ }^{*} \mathrm{p}<0.05 ;{ }^{* *} \mathrm{p}<0.01\right)$ between the $\mathrm{HD}$ group and healthy controls are indicated

\begin{tabular}{|c|c|c|c|c|c|}
\hline \multirow[t]{2}{*}{ Muscle action } & \multirow[t]{2}{*}{ Side } & \multicolumn{2}{|r|}{ Healthy control group } & \multicolumn{2}{|c|}{ HD compared to control group } \\
\hline & & \multicolumn{2}{|c|}{ Mean (SD) maximal strength scores $(\mathrm{N})$} & $\begin{array}{l}\text { Z-scores for } \\
\text { HD group }\end{array}$ & $\begin{array}{l}95 \% \mathrm{Cl} \text { of difference } \\
\text { for strength scores }\end{array}$ \\
\hline \multirow[t]{2}{*}{ Hip extension } & Right & $56.3(48.3)^{* *}$ & $134.6(49.5)$ & -1.58 & $46.9-109.6$ \\
\hline & Left & $52.5(42.8)^{* *}$ & $141.0(46.8)$ & -1.9 & $59.78-117.2$ \\
\hline \multirow[t]{2}{*}{ Hip abduction } & Right & $56.2(44.3)^{* *}$ & $124.5(33.8)$ & -2.0 & $43.1-93.5$ \\
\hline & Left & $52.2(40.6)^{* *}$ & $121.8(38.4)$ & -1.8 & $44.3-94.8$ \\
\hline \multirow[t]{2}{*}{ Knee extension } & Right & $96.2(32.1)^{* *}$ & $181.7(65.3)$ & -1.3 & $52.2-118.9$ \\
\hline & Left & $95.5(27.1)^{* *}$ & $166.5(61.9)$ & -1.2 & $39.9-102.1$ \\
\hline \multirow[t]{2}{*}{ Knee flexion } & Right & $77.3(34.4)^{* *}$ & $120.9(39.6)$ & -1.1 & $19.8-67.3$ \\
\hline & Left & $79.2(36.9)^{*}$ & $108.8(39.8)$ & -0.7 & $5.1-54.2$ \\
\hline \multirow[t]{2}{*}{ Ankle dorsiflexion } & Right & $64.0(33.5)^{* *}$ & $129.7(35.4)$ & -1.9 & $43.7-87.8$ \\
\hline & Left & $58.7(30.2)^{* *}$ & $129.4(33.5)$ & -2.1 & $50.2-91.1$ \\
\hline \multirow[t]{2}{*}{ Ankle plantarflexion } & Right & $43.9(37.2)^{* *}$ & $92.2(33.2)$ & -1.5 & $25.7-70.8$ \\
\hline & Left & $38.7(32.5)^{* *}$ & $107.4(42.8)$ & -1.6 & $44.48-93.0$ \\
\hline \multirow[t]{2}{*}{ Leg strength index } & Right & $530.9(244.5)^{* *}$ & $1053.7(288.6)$ & -1.8 & $351.5-693.9$ \\
\hline & Left & $506.1(227.2)^{* *}$ & $1050.9(293.0)$ & -1.9 & $376.9-712.6$ \\
\hline
\end{tabular}


Table 4 Spearman's correlation coefficients ( $r s$ ) between UHDRS scores and strength scores (HD group only). Significant correlations are indicated $\left.{ }^{*} p<0.05 ;{ }^{* *} p<0.01\right)$

\begin{tabular}{llllll}
\hline Muscle action & Side & $\begin{array}{l}\text { UHDRS } \\
\text { motor } \\
\text { scores }\end{array}$ & $\begin{array}{l}\text { UHDRS total } \\
\text { functional } \\
\text { capacity }\end{array}$ & $\begin{array}{l}\text { UHDRS } \\
\text { functional } \\
\text { assessment scale }\end{array}$ & $\begin{array}{l}\text { UHDRS } \\
\text { independence } \\
\text { scale }\end{array}$ \\
\hline Hip extension & Right & $-0.62^{* *}$ & $0.69^{* *}$ & $0.74^{* *}$ & $0.71^{* *}$ \\
& Left & $-0.70^{* *}$ & $0.71^{* *}$ & $0.72^{* *}$ & $0.69^{* *}$ \\
Hip abduction & Right & $-0.67^{* *}$ & $0.65^{* *}$ & $0.69^{* *}$ & $0.65^{* *}$ \\
& Left & $-0.74^{* *}$ & $0.66^{* *}$ & $0.69^{* *}$ & $0.68^{* *}$ \\
Knee extension & Right & -0.03 & 0.03 & 0.16 & -0.06 \\
Knee flexion & Left & -0.14 & 0.12 & 0.21 & 0.05 \\
& Right & -0.15 & 0.41 & 0.45 & 0.35 \\
Ankle dorsiflexion & Left & -0.14 & 0.36 & 0.393 & 0.295 \\
& Right & -0.06 & 0.06 & 0.08 & -0.03 \\
Ankle plantarflexion & Left & -0.14 & 0.20 & 0.25 & 0.13 \\
& Right & $-0.51^{*}$ & $0.59^{*}$ & $0.59^{*}$ & $0.54^{*}$ \\
Leg strength index & Left & $-0.49^{*}$ & $0.57^{*}$ & $0.59^{*}$ & $0.58^{*}$ \\
& Right & $-0.56^{* *}$ & $0.62^{* *}$ & $0.64^{* *}$ & $0.56^{*}$ \\
& Left & $-0.60^{* *}$ & $0.61^{* *}$ & $0.64^{* *}$ & $0.56^{*}$ \\
\hline
\end{tabular}

HD and is also able to detect strength differences between people with HD and a matched control group. Highest reliability with lower associated variability was identified for the leg strength indices. These indices are important when considering the relationships of muscle strength to function as they allow for the relative contribution of individual muscle groups to a total strength score and gives an overall representation of limb strength [36].

Significantly greater variability relative to a healthy control group was identified for ankle dorsi-flexion muscles; this may be a result of the pragmatic testing position utilised or the technique of hand-held dynamometry. Testing a larger sample using a fixed dynamometer might help in evaluating this particular finding further.

The reliability of the hand-held dynamometer has been assessed for use in a range of people with neurological conditions $[8,17,23,34]$ however to our knowledge, not previously in people with HD. The current study shows that despite the complex nature of HD, the hand-held dynamometer is reliable for use in this population.

People with HD were significantly weaker relative to a matched control group. This was reflected both in the individual muscle groups as well as by the strength index and the standardised Z-scores. This gives an indication of the known-groups validity of hand-held dynamometry. Significant correlations were identified between functional scores and strength indices which gives a further indication of the validity of such a measure.

Muscle strength reduction in HD has been previously implied. Mitochondrial dysfunction and morphological dysfunction of neuromuscular junctions, potentially impacting muscle strength, has been found in HD [3, 22]. The effects of creatine supplementation in people with HD were studied over the period of 1 year [31]. A 5 $-10 \%$ strength reduction over time in people with HD was measured for elbow flexors but a healthy control group was not studied and therefore it is difficult to say whether there were any strength deficits in the first place. Grip force variability has also been suggested as a measure of disease progression in HD [14, 21]. These studies have both focussed only on the upper limb (grip force and finger tip forces) and one of these did not include measurements representing a healthy control group [21]. We present data for all the lower limb muscle groups tested with a clinically useful measurement device, including a matched for age, height, weight and gender healthy control group.

This study provides reliable values of muscle strength of people with HD compared to age matched healthy subjects, but the current study may underestimate the muscle strength of the healthy subjects when the data obtained is compared to published reference data [6]. Specifically the values obtained for the plantar flexors are very low considering the weight-bearing function and the powerful nature of the muscle group acting over a very short lever arm. The plantar flexors were tested in a non-weight-bearing gravity eliminated position with the knee positioned in flexion. This means that the contribution of the gastrocnemius muscle to the recorded output may have been limited and in part may explain the low values recorded. There are limited studies examining ankle plantar-flexors [28] and do not appear to be reported in hand-held dynamometry studies $[6,34]$. This may be due to the technical difficulties experienced in standardising testing positions for this muscle group. 
Differences from the published literature may be explained by different testing protocols and devices used. There is a potential ceiling effect related to strength testing using hand-held dynamometry and there are further limitations due to stable position of the device, discomfort of the subject and the strength of the tester applying resistance with the hand-held dynamometer [32]. These latter issues could impact upon the data for healthy matched control subjects more than that of weaker people with HD with the resulting potential to underestimate strength differences.

People with HD were significantly weaker than age and gender matched control subjects. We suggest that decline in muscle strength in people with HD may be as a result of multiple components. Alteration of muscle tone seen in HD may lead to loss of motor unit activation, changes in the recruitment ordering and firing rate as proposed in stroke [25]. These changes may impair ability to produce a maximum voluntary muscle contraction effectively and consistently in people with HD, hence leading to the suggestion of increased variability [21]. This variability has been represented by the coefficient of variation in this study but the data did not confirm increased variability of lower limb measurements in people with HD relative to healthy controls, thus making this sole explanation for weakness unlikely. Whether the documented weakness is a primary manifestation of a pathological process relating to HD within muscle or is secondary to altered motor unit activation processes or to muscle wasting consequent on changed patterns of mobility or muscle usage is currently unclear.

Muscle weakness may be an important but under-reported clinical feature in HD contributing to disability and muscle strengthening interventions could be considered relevant for this patient group. Improvement of muscle strength in people with $\mathrm{HD}$ due to muscle strengthening interventions have been documented in small scale uncontrolled studies [18] although focus is usually on balance and postural impairments [19]. Loss of muscle strength in parallel with the other symptoms of the disease may contribute to physical inactivity. Lack of physical activity as a potential factor in muscle weakness has been discounted as a factor in the previous study of mitochondrial dysfunction [24], although in this case the researchers did not carry out in-depth monitoring of physical activity but relied on self report to determine physical status. Long-term communitybased activity monitoring [9] should be used to provide evidence of associated levels of physical activity. There is evidence of a relationship between decreased activity and worsening symptoms especially voluntary movements as measured by wrist-worn activity monitors [30].

Physical inactivity in parallel with the aforementioned issues may further induce plastic changes to muscles such as substantial atrophy in muscles that play an anti-gravity role, fiber-type transformations and myofibril protein loss [29]. In this situation, strength loss would be generalized to all the inactive muscles. In this study, the loss of muscle strength was not restricted to selected muscle groups of the lower limbs; the pattern of weakness was non-specific. This lends support to physical inactivity as being one of a range of potential mechanisms of the reported weakness. Further in-depth investigations using robust strength testing measures and neurophysiological techniques including twitch interpolation to test muscle activation processes alongside molecular investigations may help elucidate the cause of weakness.

Conflict of interest The authors declare no conflict of interest.

Acknowledgements The researchers would like to acknowledge the Physiotherapy Research Society for assistance with funding of travel expenses related to this study. The European Huntington's Disease Network (EHDN) and in particular Kathy Price, Jenny Naji and Olivia Handley are acknowledged for their assistance with the 'Registry' database and recruitment of people with HD. We would also like to acknowledge The Wellcome Trust Clinical Research Facility for the use of ward space for data collection and finally the participants and their family for agreeing to participate.

\section{References}

1. Andres PL, Hedlund W, Finison L, Conlon T, Felmus M, Munsat TL (1986) Quantitative motor assessment in amyotrophic lateral sclerosis. Neurology 36:937-941

2. Andrews AW, Bohannon RW (2000) Distribution of muscle strength impairments following stroke. Clin Rehabil 14:79-87

3. Arenas J, Campos Y, Ribacoba R, Martin MA, Rubio JC, Ablanedo P, Cabello A (1998) Complex I defect in muscle from patients with Huntington's disease. Ann Neurol 43:397-400
4. Batterham A, George K (2003) Reliability in evidences based clinical practice: a primer for allied healthy professions. Physical Therapy in Sport 4:122-128

5. Bland JM (2000) An introduction to medical statistics. Oxford University Press, Oxford

6. Bohannon RW (1997) Reference values for extremity muscle strength obtained by hand-held dynamometry from adults aged 20 to 79 years. Arch Phys Med Rehabil 78:26-32
7. Bohannon RW (2006) Reference values for the timed up and go test: a descriptive meta-analysis. J Geriatr Phys Ther 29:64-68

8. Bohannon RW (1986) Test-retest reliability of hand-held dynamometry during a single session of strength assessment. Phys Ther 66:206-209

9. Busse ME, Pearson OR, Van Deursen R, Wiles CM (2004) Quantified measurement of activity provides insight into motor function and recovery in neurological disease. J Neurol Neurosurg Psychiatry 75:884-888 
10. Cronbach LJ, Meehl PE (1955) Construct validity in psychological tests. Psychol Bull 52:281-302

11. Donner A, Eliasziw M (1987) Sample size requirements for reliability studies. Stat Med 6:441-448

12. Faul F, Erdfelder E, Lang AG, Buchner A (2007) $G^{*}$ Power 3: a flexible statistical power analysis program for the social, behavioral, and biomedical sciences. Behav Res Methods 39:175-191

13. Goonetilleke A, Modarres-Sadeghi H, Guiloff RJ (1994) Accuracy, reproducibility, and variability of hand-held dynamometry in motor neuron disease. J. Neurol. Neurosurg. Psychiatry 57:326-332

14. Gordon AM, Quinn L, Reilmann R, Marder K (2000) Coordination of prehensile forces during precision grip in Huntington's disease. Exp Neurol 163:136-148

15. Hobart JC, Lamping DL, Thompson AJ (1996) Evaluating neurological outcome measures: the bare essentials. J Neurol Neurosurg Psychiatry 60: 127-130

16. Kilmer DD, McCrory MA, Wright N, Rosko R, Kim H, Aitkens S (1997) Hand-held dynamometry reliability in persons with neuropathic weakness. Arch Phys Med Rehabil 78:1364-1368

17. Nollet F, Beelen A (1999) Strength assessment in postpolio syndrome: validity of a hand-held dynamometer in detecting change. Arch Phys Med Rehabil 80:1316-1323

18. Peacock IW (1987) A physical therapy program for Huntington's disease patients. Clinical Management in Physical Therapy 7:22-23

19. Quinn L, Rao A (2002) Physical therapy for people with Huntington disease: current perspectives and case report. Neurology Report 26(3): 145-153
20. Quinn N, Brown R, Craufurd D, Goldman S, Hodges J, Kieburtz K, Lindvall O, MacMillan J, Roos R (1996) Core Assessment Program for Intracerebral Transplantation in Huntington's Disease (CAPIT-HD). Mov Disord 11: 143-150

21. Reilmann R, Kirsten F, Quinn L, Henningsen $\mathrm{H}$, Marder K, Gordon AM (2001) Objective assessment of progression in Huntington's disease: a 3-year follow-up study. Neurology 57:920-924

22. Ribchester RR, Thomson D, Wood NI, Hinks T, Gillingwater TH, Wishart TM, Court FA, Morton AJ (2004) Progressive abnormalities in skeletal muscle and neuromuscular junctions of transgenic mice expressing the Huntington's disease mutation. Eur J Neurosci 20: 3092-3114

23. Riddle DL, Finucane SD, Rothstein JM, Walker ML (1989) Intrasession and intersession reliability of hand-held dynamometer measurements taken on brain-damaged patients. Phys Ther 69: 182-194

24. Saft C, Zange J, Andrich J, Muller K, Lindenberg K, Landwehrmeyer B, Vorgerd M, Kraus PH, Przuntek H, Schols L (2005) Mitochondrial impairment in patients and asymptomatic mutation carriers of Huntington's disease. Mov Disord 20:674-679

25. Shepherd RB (2001) Exercise and training to optimize functional motor performance in stroke: driving neural reorganization? Neural Plast 8:121-129

26. Shrout P, Fleiss J (1979) Intraclass correlation: Uses in assessing rater reliability. Psychol Bull 86:420-426

27. Sim J, Arnell P (1993) Measurement validity in physical therapy research. PhysTher 73:102-110

28. Simoneau G, Ulbrecht D, Derr J, Cavanagh P (1995) Role of somatosensory input in the control of human posture. Gait \& Posture 3:115-122
29. Thompson LV (1994) Effects of age and training on skeletal muscle physiology and performance. Phys Ther 74:71-81

30. van Vugt JP, Siesling S, Piet KK, Zwinderman AH, Middelkoop HA, van Hilten JJ, Roos RA (2001) Quantitative assessment of daytime motor activity provides a responsive measure of functional decline in patients with Huntington's disease. Mov Disord 16:481-488

31. Verbessem P, Lemiere J, Eijnde BO, Swinnen S, Vanhees L, Van Leemputte M, Hespel P, Dom R (2003) Creatine supplementation in Huntington's disease: a placebo-controlled pilot trial. Neurology 61(7):925-930

32. Wadsworth CT, Krishnan R, Sear M, Harrold J, Nielsen DH (1987) Intrarater reliability of manual muscle testing and hand-held dynametric muscle testing. Phys Ther 67:1342-1347

33. Wiles CM, Busse ME, Sampson CM, Rogers MT, Fenton-May J, van Deursen R (2006) Falls and stumbles in myotonic dystrophy. J Neurol Neurosurg Psychiatry 77:393-396

34. Wiles CM, Karni Y (1983) The measurement of muscle strength in patients with peripheral neuromuscular disorders. J Neurol Neurosurg Psychiatry 46:1006-1013

35. Wiles CM, Karni Y, Nicklin J (1990) Laboratory testing of muscle function in the management of neuromuscular disease. J Neurol Neurosurg Psychiatry 53:384-387

36. Willen C, Sunnerhagen KS, Ekman C, Grimby G (2004) How is walking speed related to muscle strength? A study of healthy persons and persons with late effects of polio. Arch Phys Med Rehabil 85:1923-1928 